\title{
Magnetic Resonance Imaging Findings Differentiating Cutaneous Basal Cell Carcinoma from Squamous Cell Carcinoma in the Head and Neck Region
}

\author{
Masaya Kawaguchi, MD' ${ }^{1}$, Hiroki Kato, $M D^{1}$, Hiroyuki Tomita, $M D^{2}$, Akira Hara, MD², Natsuko Suzui, MD², \\ Tatsuhiko Miyazaki, MD³, Kanako Matsuyama, MD4, Mariko Seishima, MD ${ }^{4}$, Masayuki Matsuo, MD ${ }^{1}$ \\ Departments of ${ }^{1}$ Radiology, ${ }^{2}$ Tumor Pathology, ${ }^{3}$ Pathology, and ${ }^{4}$ Dermatology, Gifu University School of Medicine, Gifu, Japan
}

Objective: This study aimed to evaluate the efficacy of magnetic resonance (MR) imaging in differentiating between cutaneous basal cell carcinoma (CBCC) and cutaneous squamous cell carcinoma (CSCC) in the head and neck region.

Materials and Methods: Among patients with cutaneous head and neck cancers, 14 with primary $\mathrm{CBCCs}$ and 15 with primary CSCCs with a histologic tumor height of $\geq 4 \mathrm{~mm}$ underwent MR examinations; the findings were then examined for correlations. Results: CBCCs $(71 \%)$ occurred more frequently on the nose than CSCCs $(13 \%)(p<0.01)$. The maximum diameter $(23.5 \pm 7.2$ $\mathrm{mm}$ vs. $12.7 \pm 4.5 \mathrm{~mm} ; p<0.01)$ and diameter-to-height ratio $(2.8 \pm 0.9$ vs. $1.7 \pm 0.4 ; p<0.01)$ were significantly greater in cSCCs than in CBCCs. Superficial ulcer formation $(67 \%$ vs. $21 \% ; p<0.05)$, protrusion into the subcutaneous tissue $(60 \%$ vs. $21 \% ; p<0.05)$, ill-demarcated deep tumor margins ( $60 \%$ vs. $7 \% ; p<0.01)$, and peritumoral fat stranding $(93 \%$ vs. $7 \%$; $p<0.01)$ were more frequently observed in cSCCs than in CBCCs. Intratumoral T2-hyperintense foci $(57 \%$ vs. $13 \% ; p<0.05)$ were more frequently observed in $\mathrm{CBCCs}$ than in CSCCs.

Conclusion: $\mathrm{CBCCS}$ predominantly occurred on the nose with intratumoral T2-hyperintense foci, whereas CSCCs predominantly exhibited a flattened configuration, superficial ulcer formation, protrusion into the subcutaneous tissue, ill-demarcated deep tumor margin, and peritumoral fat stranding.

Keywords: Basal cell carcinoma; Squamous cell carcinoma; Skin cancer; Head and neck; MRI

\section{INTRODUCTION}

Cutaneous basal cell carcinoma ( $\mathrm{CBCC})$ is the most common histologic subtype of skin cancer, which is derived from the basal cells of the interfollicular epidermis and/ or hair follicles. CBCCs most commonly occur during the seventh decade of life and affect men more frequently than women. The incidence rates of $\mathrm{CBCC}$ are related to

Received July 8, 2019; accepted after revision November 4, 2019. Corresponding author: Hiroki Kato, MD, Department of Radiology, Gifu University School of Medicine, 1-1 Yanagido, Gifu 501-1194, Japan.

- Tel: (8158) 230-6439 - Fax: (8158) 230-6440

- E-mail: hkato@gifu-u.ac.jp

This is an Open Access article distributed under the terms of the Creative Commons Attribution Non-Commercial License (https://creativecommons.org/licenses/by-nc/4.0) which permits unrestricted non-commercial use, distribution, and reproduction in any medium, provided the original work is properly cited. the geographical latitude and are higher in lighter-skinned populations. Given the abundant evidence supporting the role of ultraviolet radiation exposure as the most important risk factor for $\mathrm{CBCC}$ carcinogenesis, $\mathrm{CBCC}$ predominantly occur on the exposed parts of the body, with $75-85 \%$ of lesions found in the head and neck regions (1-4).

Cutaneous squamous cell carcinoma (CSCC) is the second most common type of skin cancer derived from epidermal keratinocytes exhibiting various degrees of differentiation. cSCCs predominantly occur during the eighth decade of life and are more common in men than women. Moreover, the highest incidence rates have been observed among white populations with fair complexion, blue eyes, and blonde or red hair. Considering that the risk of developing CSCC depends on the lifetime accumulation of ultraviolet radiation damage, predilection sites for $\mathrm{CSCC}$ include chronically exposed areas, such as the face (particularly the lips, ears, nose, cheeks, and eyelids) and dorsum of the 
hands $(4,5)$.

Although the metastasis rate of $\mathrm{CBCC}$ ranges from $0.0016 \%$ to $0.1343 \%$ (6), approximately $1.9-4.9 \%$ of cSCCs metastasize to regional lymph nodes or more distant sites (7-9). According to the National Comprehensive Cancer Network (NCCN) risk stratification, the recommended surgical margins for uninvolved skin around the tumor and/or biopsy site to a depth reaching mid-subcutaneous adipose tissue for standard excision was $4 \mathrm{~mm}$ in low-risk CBCCs (10) and 4-6 mm in low-risk cSCCs (11). In addition, for non-surgical candidates, the NCCN clinical practice guidelines recommend radiotherapy alone for $\mathrm{CBCCs}$ but combination chemoradiation therapy for $\operatorname{cSCCs}(10,11)$. Therefore, preoperative differentiation between $\mathrm{CBCC}$ and CSCC is essential for establishing appropriate decisions regarding the treatment strategy. Although a few studies have already described the imaging findings of CSCCs and cBCCs (12-16), to the best of our knowledge, no study has reported detailed magnetic resonance (MR) imaging findings that differentiate $\mathrm{CBCCs}$ from CSCCs. Hence, the present study aimed to assess MR imaging characteristics that can be used to differentiate $\mathrm{CBCCs}$ from $\mathrm{CSCC}$ in the head and neck region.

\section{MATERIALS AND METHODS}

\section{Patients}

The study was approved by the human research committee of the Institutional Review Board of Gifu University (2019-126) and complied with the guidelines of the Health Insurance Portability and Accountability Act. The requirement for informed consent was waived due to the retrospective nature of this study. The electronic medical chart system of our University Hospital was searched for patients with histopathologically proven $\mathrm{CBCCs}$ and $\mathrm{CSCCs}$ in the head and neck region between January 2010 and December 2018. Among the identified patients, 21 with CBCCs and 16 with CSCCs underwent preoperative MR imaging, while seven with $\mathrm{CBCCS}$ and one with $\mathrm{CSCC}$ sere excluded given that they had a histological tumor height of $<4 \mathrm{~mm}$, recurrent tumors, or inappropriate MR images. A total of 14 patients with primary $\mathrm{CBCCS}$ (eight men and six women; age range, 39-88 years; median age, 72 years) and 15 with primary CSCCS (11 men and four women; age range, 46-88 years; median age, 82 years) were included in this study.

\section{MR Imaging}

MR imaging was performed using a 1.5T MR imaging system (Intera Achieva 1.5T Pulsar; Philips Medical Systems, Best, The Netherlands) for seven CBCCs and seven CSCCs or a 3T MR imaging system (Intera Achieva 3.0T Quasar Dual; Philips Medical Systems) for seven CBCCs and eight cSCCs. MR imaging data were obtained using the parallel imaging technique at a section thickness of 3-4 mm with a 1-mm intersection gap, and a $16 \times 16-20 \times 20-\mathrm{cm}$ field of view. Axial T1-weighted spin-echo (repetition time [TR]/ echo time [TE], 387-752/9-16 ms), axial and oblique sagittal or coronal T2-weighted fast spin-echo (TR/TE, 3000-5982/90-120 ms), and axial and oblique sagittal or coronal fat-suppressed T2-weighted fast spin-echo (TR/TE, 3030-6123/80-120 ms) images were obtained for all 29 patients.

\section{Imaging Data Analysis}

Two radiologists with 20 and 6 years of posttraining experience, respectively, in head and neck imaging independently reviewed all MR images, and any disagreements were resolved by consensus. The reviewers were unaware of the clinical findings and pathological diagnoses.

First, the reviewers noted the site of tumor occurrence, measured the maximum diameter and height of the lesion on T2-weighted images, and then calculated the diameterto-height ratio.

Second, the reviewers evaluated the marginal characteristics of the tumors. They assessed the presence of superficial ulceration and protrusion into adjacent subcutaneous tissue on T2-weighted images. Deep tumor margins between the tumors and adjacent subcutaneous tissue were assessed on T2-weighted images and then classified into either ill-demarcated or well-demarcated. The presence of peritumoral fat stranding, which indicates increased signal intensity of the surrounding soft tissue, was assessed on fat-suppressed T2-weighted images.

Third, the reviewers evaluated the internal characteristics of the tumors. They assessed the presence and number of intratumoral T2-hyperintense foci, which were defined as well-demarcated, markedly hyperintense areas equivalent in intensity to the cerebrospinal fluid on fat-suppressed T2-weighted images. Intratumoral homogeneity was assessed using T2-weighted images, excluding intratumoral T2-hyperintense foci, and were classified as either homogeneous or heterogeneous. 


\section{Statistical Analysis}

All statistical analyses were performed using SPSS version 22.0 (IBM Corp., Armonk, NY, USA). The chi-square test or Fisher's exact test was performed to compare the frequencies of qualitative assessments (occurrence site, superficial ulceration, protrusion into subcutaneous tissue, deep tumor margins, peritumoral fat stranding, intratumoral T2-hyperintense foci, and intratumoral homogeneity).

The unpaired $t$ test was used to compare the quantitative measurements (maximum diameter and height of the lesion, diameter-to-height ratio, and number of intratumoral T2hyperintense foci). Interobserver variability of qualitative assessments was assessed using kappa statistics. $P$ values < 0.05 were considered significant.

\section{RESULTS}

The qualitative imaging findings are summarized in Table 1. CBCCs were located on the nose in 10 patients, eyelid in three, and auricle in one. Meanwhile, cSCCs were located on the cheek in five patients, scalp in three, nose in two, auricle in two, forehead in two, and mandible in one. cBCCs $(71 \%)$ more frequently occurred on the nose than $\operatorname{cSCCs}(13 \%)(p<0.01)$.

The quantitative measurements are summarized in Table 2. The maximum diameter $(23.5 \pm 7.2 \mathrm{~mm}$ vs. 12.7 $\pm 4.5 \mathrm{~mm} ; p<0.01)$ and diameter-to-height ratio $(2.8 \pm$ 0.9 vs. $1.7 \pm 0.4 ; p<0.01)$ were significantly greater in CSCCs than in CBCCs (Figs. 1, 2). However, no significant difference in height $(8.9 \pm 3.5 \mathrm{~mm}$ vs. $7.7 \pm 3.5 \mathrm{~mm} ; p=$ 0.208 ) was observed between CSCCs and CBCCs.

Superficial ulcer formation ( $67 \%$ vs. $21 \% ; p<0.05)$, protrusion into subcutaneous tissue $(60 \%$ vs. $21 \% ; p<$ $0.05)$, ill-demarcated deep tumor margins ( $60 \%$ vs. $7 \% ; p<$ $0.01)$, and peritumoral fat stranding $(93 \%$ vs. $7 \% ; p<0.01)$ were more frequently observed in CSCCs than in $\mathrm{CBCCs}$ (Figs. 1,2).

The intratumoral T2-hyperintense foci (57\% vs. $13 \%$; $p<$ 0.05 ) were more frequently observed in $\mathrm{CBCCs}$ than in $\mathrm{CSCCs}$ (Figs. 1, 2). More than three intratumoral T2-hyperintense foci were observed in 6 of $14 \mathrm{CBCCs}(43 \%)$, whereas they were not seen in CSCCs. However, no significant differences in the number of intratumoral T2-hyperintense foci ( $1.5 \pm 0.7$ vs. $8.9 \pm 9.1 ; p=0.063)$ and intratumoral heterogeneous signal intensity ( $60 \%$ vs. $29 \% ; p=0.089$ ) were observed between cSCCs and CBCCs.

The kappa values for the two observers with regard to evaluating superficial ulcer formation, protrusion into subcutaneous tissue, ill-demarcated deep tumor margins, peritumoral fat stranding, and intratumoral heterogeneous signal intensity showed substantial agreement $(0.72,0.79$, $0.64,0.72$, and 0.72 , respectively), and that with regard to evaluating the intratumoral T2-hyperintense foci showed excellent agreement (1.00).

\section{DISCUSSION}

Predilection sites for $\mathrm{CBCCs}$ and $\mathrm{CSCC}$ s include the head and neck, given that such regions are more frequently exposed to ultraviolet radiation. The present study revealed

\section{Table 2. Quantitative Measurements of $\mathrm{CBCC}$ and $\mathrm{CSCC}$}

\begin{tabular}{lccc}
\hline \multicolumn{1}{c}{ Quantitative Measurements } & $\begin{array}{c}\mathrm{CBCC} \\
(\mathrm{n}=14)\end{array}$ & $\begin{array}{c}\mathrm{CSCC} \\
(\mathrm{n}=15)\end{array}$ & $P$ \\
\hline Maximum diameter $(\mathrm{mm})$ & $12.7 \pm 4.5$ & $23.5 \pm 7.2$ & $<0.001^{*}$ \\
Height $(\mathrm{mm})$ & $7.7 \pm 3.5$ & $8.9 \pm 3.5$ & 0.208 \\
Diameter-to-height ratio & $1.7 \pm 0.4$ & $2.8 \pm 0.9$ & $<0.001^{*}$ \\
Number of T2-hyperintense foci & $8.9 \pm 9.1$ & $1.5 \pm 0.7$ & 0.063 \\
\hline
\end{tabular}

Data are shown as mean \pm 1 standard deviation. * Significant differences in values were observed between $\operatorname{cBCC}$ and $\operatorname{CSCC}(p<$ 0.01).

Table 1. Qualitative Imaging Findings of $\mathrm{CBCC}$ and $\mathrm{CSCC}$

\begin{tabular}{|c|c|c|c|}
\hline Qualitative Imaging Findings & $\mathrm{CBCC}(\mathrm{n}=14)$ & $\operatorname{cSCC}(n=15)$ & $P$ \\
\hline Location of nose & $10(71)$ & $2(13)$ & $0.046^{*}$ \\
\hline Superficial ulcer formation & $3(21)$ & $10(67)$ & $0.014^{*}$ \\
\hline Protrusion into subcutaneous tissue & $3(21)$ & $9(60)$ & $0.035^{*}$ \\
\hline Ill-demarcated deep tumor margin & $1(7)$ & $9(60)$ & $0.004^{\dagger}$ \\
\hline Peritumoral fat stranding & $1(7)$ & $14(93)$ & $<0.001^{\dagger}$ \\
\hline Intratumoral heterogeneous signal intensity & $4(29)$ & $9(60)$ & 0.089 \\
\hline Intratumoral T2-hyperintense foci & $8(57)$ & $2(13)$ & $0.017^{*}$ \\
\hline
\end{tabular}

Data are expressed as numbers of patients, and numbers in parentheses are frequencies expressed as percentages. *Significant differences in frequencies were observed between $\mathrm{CBCC}$ and $\operatorname{cSCC}(p<0.05)$, 'Significant differences in frequencies were observed between $\mathrm{CBCC}$ and $\operatorname{cSCC}(p<0.01) \cdot c B C C=$ cutaneous basal cell carcinoma, $\mathrm{CSCC}=$ cutaneous squamous cell carcinoma 

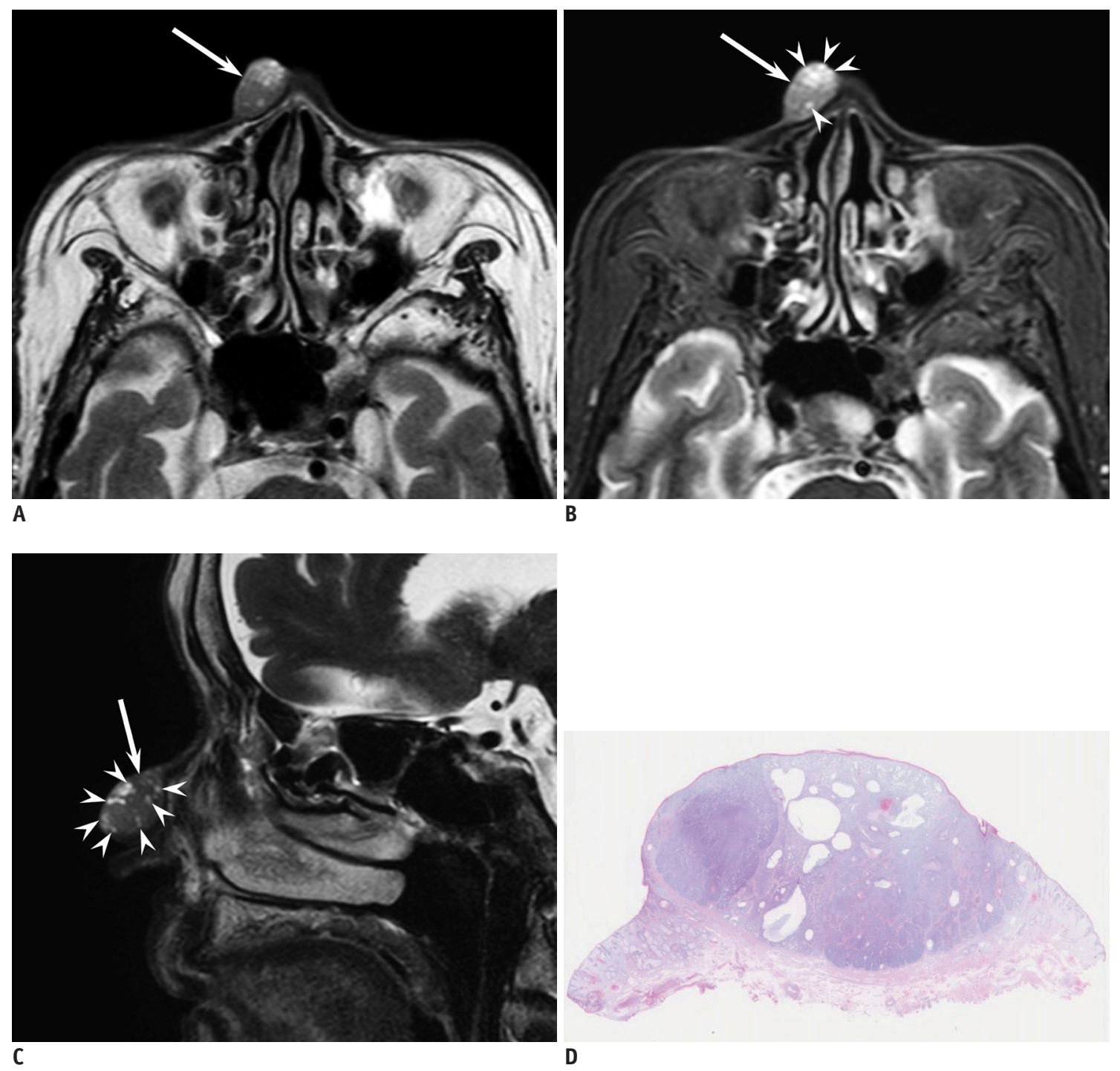

Fig. 1. 87-year-old woman with cutaneous basal cell carcinoma of right nose.

A. Axial T2-weighted image (TR/TE, 3000/90 ms) showing well-demarcated, elliptic, cutaneous lesion (arrow) without superficial ulcer formation and protrusion into subcutaneous tissue. B. Axial fat-suppressed T2-weighted image (TR/TE, 3290/80 ms) showing T2-hyperintense foci (arrowheads) within cutaneous lesion (arrow); peritumoral fat stranding is not observed. C. Sagittal fat-suppressed T2-weighted image (TR/TE, 4350/120 ms) clearly showing T2-hyperintense foci (arrowheads) within cutaneous lesion (arrow). D. Histological specimen (H\&E stain, x 2.5) showing well-demarcated mass in dermis with multiple cystic cavities filled with mucinous contents. $\mathrm{H \& E}=$ hematoxylin and eosin, $\mathrm{TE}=$ echo time, $T R=$ repetition time

that $\mathrm{CBCCs}(71 \%)$ were more frequently found on the nose than CSCCs (13\%). Consistent with our results, other studies have also shown that the most common anatomical site for CBCCs was the nose $(32-49 \%)(2,17)$. In contrast, studies have also shown that cSCCS usually occur on the lateral aspect of the face, including the auricle, and auricular, buccal, parotid-masseteric, and temporal areas; therefore, only $5-14 \%$ of cSCCs occur on the nose $(17,18)$. Differences in the distribution between CBCCs and CSCCs can thus be a clinically useful method for differentiating between both pathologies.
CBCCs have a variety of histopathological variants that always contain aggregates of basaloid cells with scant cytoplasm, as well as hyperchromatic nuclei surrounded by fibromyxoid stromal changes that induce retraction of the tumor from the stroma. Nodular $\mathrm{CBCC}$, the most common variant, is characterized by large tumor nodules in the dermis, whereas infiltrating $\mathrm{CBCC}$ is characterized by narrow tumor cords and nests with an irregular, infiltrating growth pattern. Among the $14 \mathrm{CBCC}$ included in this study, 13 $(93 \%)$ were nodular with well-demarcated deep tumor margins, whereas the remaining one $(7 \%)$ infiltrated into 

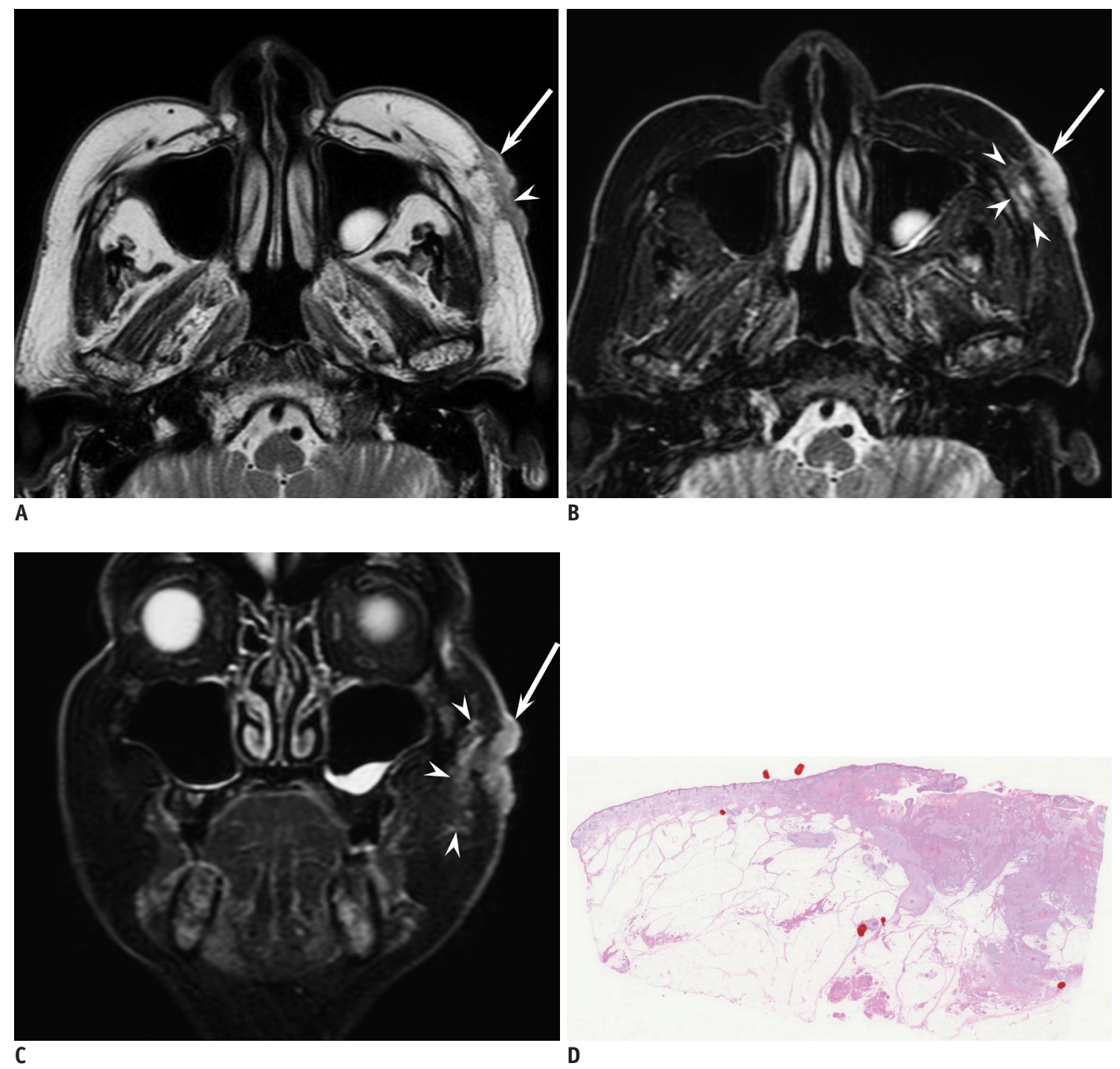

Fig. 2. 77-year-old man with cutaneous squamous cell carcinoma of left cheek.

A. Axial T2-weighted image (TR/TE, 4100/90 ms) showing ill-demarcated, flattened, cutaneous lesion (arrow) with superficial ulcer formation (arrowhead) and protrusion into subcutaneous tissue. B. Axial fat-suppressed T2-weighted image (TR/TE, 3636/90 ms) showing peritumoral fat stranding (arrowheads) adjacent to cutaneous lesion (arrow); T2-hyperintense foci are not seen. C. Coronal fat-suppressed T2-weighted image (TR/TE, 4542/90 ms) clearly showing peritumoral fat stranding (arrowheads) adjacent to cutaneous lesion (arrow). D. Histological specimen (H\&E stain, $\times 2.5$ ) shows ill-demarcated mass infiltrating subcutaneous fat tissue.

the subcutaneous tissue with an ill-demarcated deep tumor margin and peritumoral fat stranding.

CSCCs usually progress from in situ lesions to progressively more deeply invasive tumors. In situ cSCCs may appear as roughened hyperkeratotic lesions mimicking benign keratosis, dermatoses, or lichen simplex chronicus. Welldifferentiated cSCCs form crateriform lesions with central keratin plugs, whereas less well-differentiated cSCCs produce irregular, erythematous scaling nodules and plaques. CSCCs are considered invasive tumors, referring to cancer cells that have developed beyond the epidermis. Therefore, protrusion into the subcutaneous tissue $(60 \%)$, ill-demarcated deep tumor margins $(60 \%)$, and peritumoral fat stranding (93\%) were frequently observed among the CSCCs included in this study. In addition, with invasion and progressive growth, hyperkeratotic nodules may develop ulceration. Malignant degeneration of untreated chronic wounds, referred to as Marjolin's ulcers, have been reported in $\operatorname{cSCCs}(19,20)$. Accordingly, superficial ulcer formation (67\%) was also frequently observed in the cSCCs included in this study. These imaging findings correspond to the aggressive and invasive nature of CSCCs.

T2-weighted imaging has shown that CBCCs exhibit markedly higher hyperintensity relative to muscle and 
moderate hyperintensity relative to skin (14). In the present study, intratumoral T2-hyperintense foci (57\%) were frequently observed in $\mathrm{CBCC}$. A previous study evaluating ultrasonographic findings reported that hypersonographic spots in CBCCs seemed to correspond to calcification, horn cysts, or clusters of apoptotic cells at the centers of $C B C C$ nests (21). However, in all eight $\mathrm{cBCC}$ s with intratumoral T2-hyperintense foci, a radiologic-pathologic correlation on a one-to-one basis was performed and revealed cystic cavities filled with mucinous contents within $\mathrm{CBCCs}$ corresponding to the intratumoral T2-hyperintense foci, an MR imaging finding that may be characteristic to $\mathrm{CBCC}$.

The majority of CSCCs have a nonspecific signal intensity pattern of hypointensity on T1-weighted images and iso- to hyperintensity on T2-weighted images $(13,19)$. However, as CSCCs progress and cystic necrosis develops within the tumor, they generate heterogeneous signal intensities, including non-enhanced regions, on contrast-enhanced images (13). The cSCCs in this study frequently exhibited heterogeneous signal intensities on T2-weighted images $(60 \%)$.

Several limitations of this study are worth noting. First, the cohort size was relatively small considering that the study was conducted at a single institution. Second, given that MR imaging could not accurately evaluate low-height tumors, only those with a histologic height of $\geq 4 \mathrm{~mm}$ were included in this study, which may have introduced a selection bias. Third, given that the CBCCs in this study were mostly nodular CBCCs $(13 / 14)$, the features distinguishing $\mathrm{CBCCs}$ from CSCCs might not reflect characteristics of infiltrating $\mathrm{CBCCS}$. Fourth, owing to the retrospective nature of this study, only five patients had undergone contrast-enhanced MR imaging. Among these five patients with tumors (three $\mathrm{CBCCS}$ and two $\mathrm{CSCCs}$ ), relatively homogeneous enhancement was observed. In addition, peritumoral fat enhancement was observed only in tumors with peritumoral fat stranding on fat-suppressed T2-weighted images. Therefore, we presume that contrastenhanced MR imaging has limited effectiveness for the differentiation between CBCCs and CSCCs. Fifth, according to NCCN guideline, imaging studies should be performed when extensive disease, such as bone involvement, perineural invasion, or deep soft tissue involvement, is suspected. As clinical diagnosis is routinely confirmed by biopsy, imagingbased differentiation is usually not required. This is the first study to reveal the differences in MR features between skin cancers; therefore, the present study may give provide recognition to the supplementary role of MR imaging in the differentiation of skin cancers.

In conclusion, $\mathrm{CBCC}$ predominantly occurred on the nose with intratumoral T2-hyperintense foci corresponding to cystic cavities filled with mucinous contents within CBCCs, an MR imaging finding of intratumoral T2-hyperintense foci that may be characteristic. Meanwhile, characteristic MR imaging findings for CSCCs included a flattened configuration, superficial ulcer formation, protrusion into subcutaneous tissue, ill-demarcated deep tumor margins, and peritumoral fat stranding, which correspond to the aggressive and invasive nature of these tumors. We emphasize that this is the first study that compared the MR imaging findings of the most common skin cancers, in an era of growing prevalence of skin cancers, as well as an aging population.

\section{Conflicts of Interest}

The authors have no potential conflicts of interest to disclose.

\author{
ORCID iDs \\ Hiroki Kato \\ https://orcid.org/0000-0001-5926-1895 \\ Masaya Kawaguchi \\ https://orcid.org/0000-0002-2807-0524 \\ Hiroyuki Tomita \\ https://orcid.org/0000-0002-3291-0274 \\ Akira Hara \\ https://orcid.org/0000-0002-5554-8060 \\ Natsuko Suzui \\ https://orcid.org/0000-0002-3593-8432 \\ Tatsuhiko Miyazaki \\ https://orcid.org/0000-0002-4750-2046
}

\section{REFERENCES}

1. Demirseren DD, Ceran C, Aksam B, Demirseren ME, Metin A. Basal cell carcinoma of the head and neck region: a retrospective analysis of completely excised 331 cases. J Skin Cancer 2014;2014:858636

2. Janjua OS, Qureshi SM. Basal cell carcinoma of the head and neck region: an analysis of 171 cases. J Skin Cancer 2012;2012:943472

3. Nakayama M, Tabuchi K, Nakamura Y, Hara A. Basal cell carcinoma of the head and neck. J Skin Cancer 2011;2011:496910

4. Narayanan DL, Saladi RN, Fox JL. Ultraviolet radiation and 
MR Findings Differentiating Cutaneous BCC from SCC in the Head and Neck

skin cancer. Int J Dermatol 2010;49:978-986

5. Gurudutt VV, Genden EM. Cutaneous squamous cell carcinoma of the head and neck. J Skin Cancer 2011;2011:502723

6. Nguyen-Nielsen M, Wang L, Pedersen L, Olesen AB, Hou $\mathrm{J}$, Mackey $\mathrm{H}$, et al. The incidence of metastatic basal cell carcinoma (mBCC) in Denmark, 1997-2010. Eur J Dermatol 2015;25:463-468

7. Joseph MG, Zulueta WP, Kennedy PJ. Squamous cell carcinoma of the skin of the trunk and limbs: the incidence of metastases and their outcome. Aust N Z J Surg 1992;62:697701

8. Brantsch KD, Meisner C, Schönfisch B, Trilling B, WehnerCaroli J, Röcken $M$, et al. Analysis of risk factors determining prognosis of cutaneous squamous-cell carcinoma: a prospective study. Lancet Oncol 2008;9:713-720

9. Brougham ND, Dennett ER, Cameron R, Tan ST. The incidence of metastasis from cutaneous squamous cell carcinoma and the impact of its risk factors. J Surg Oncol 2012;106:811-815

10. Work Group; Invited Reviewers, Kim JYS, Kozlow JH, Mittal B, Moyer J, Olencki T, Rodgers P. Guidelines of care for the management of basal cell carcinoma. J Am Acad Dermatol 2018;78:540-559

11. Work Group; Invited Reviewers, Kim JYS, Kozlow JH, Mittal B, Moyer J, Olenecki T, Rodgers P. Guidelines of care for the management of cutaneous squamous cell carcinoma. J Am Acad Dermatol 2018;78:560-578

12. Baheti AD, Tirumani SH, Giardino A, Rosenthal MH, Tirumani $\mathrm{H}$, Krajewski $\mathrm{K}$, et al. Basal cell carcinoma: a comprehensive review for the radiologist. AJR Am J Roentgenol 2015;204: W132-W140
13. Kim JH, Kim JY, Chun KA, Jee WH, Sung MS. MR imaging manifestations of skin tumors. Eur Radiol 2008;18:2652-2661

14. Gufler H, Franke FE, Rau WS. High-resolution MRI of basal cell carcinomas of the face using a microscopy coil. AJR Am J Roentgenol 2007;188:W480-W484

15. Budak MJ, Weir-McCall JR, Yeap PM, White RD, Waugh $S A$, Sudarshan TA, et al. High-resolution microscopy-coil MR imaging of skin tumors: techniques and novel clinical applications. Radiographics 2015;35:1077-1090

16. Juan YH, Saboo SS, Tirumani SH, Khandelwal A, Shinagare AB, Ramaiya $\mathrm{N}$, et al. Malignant skin and subcutaneous neoplasms in adults: multimodality imaging with CT, MRI, and $18 \mathrm{~F}-\mathrm{FDG}$ PET/CT. AJR Am J Roentgenol 2014;202:W422-W438

17. Choi JH, Kim YJ, Kim H, Nam SH, Choi YW. Distribution of basal cell carcinoma and squamous cell carcinoma by facial esthetic unit. Arch Plast Surg 2013;40:387-391

18. Szewczyk M, Pazdrowski J, Golusiński P, Dańczak-Pazdrowska A, Marszałek S, Golusiński W. Analysis of selected risk factors for nodal metastases in head and neck cutaneous squamous cell carcinoma. Eur Arch Otorhinolaryngol 2015;272:3007-3012

19. Chiang KH, Chou AS, Hsu YH, Lee SK, Lee CC, Yen PS, et al. Marjolin's ulcer: MR appearance. AJR Am J Roentgenol 2006;186:819-820

20. Singer AD, Datir A, Tresley J, Langley T, Clifford PD, Jose J, et al. Benign and malignant tumors of the foot and ankle. Skeletal Radiol 2016;45:287-305

21. Uhara H, Hayashi K, Koga H, Saida T. Multiple hypersonographic spots in basal cell carcinoma. Dermatol Surg 2007;33:1215-1219 